\title{
Fabrication and subband gap optical properties of silicon supersaturated with chalcogens by ion implantation and pulsed laser melting
}

\author{
Brion P. Bob, ${ }^{1}$ Atsushi Kohno, ${ }^{1,2}$ Supakit Charnvanichborikarn, ${ }^{3}$ Jeffrey M. Warrender, ${ }^{4, a)}$ \\ Ikurou Umezu, ${ }^{1,5}$ Malek Tabbal, ${ }^{6}$ James S. Williams, ${ }^{3}$ and Michael J. Aziz ${ }^{1, b)}$ \\ ${ }^{1}$ Harvard School of Engineering and Applied Sciences, Cambridge, Massachusetts 02138, USA \\ ${ }^{2}$ Department of Applied Physics, Fukuoka University, Fukuoka 814-0180, Japan \\ ${ }^{3}$ Research School of Physics and Engineering, Australian National University, Canberra City ACT 2601, \\ Australia \\ ${ }^{4}$ Benet Laboratories, U.S. Army ARDEC, Watervliet, New York 12189, USA \\ ${ }^{5}$ Department of Physics, Konan University, Kobe 658-8501, Japan \\ ${ }^{6}$ Department of Physics, American University of Beirut, Beirut 1107 2020, Lebanon
}

(Received 26 October 2009; accepted 2 April 2010; published online 16 June 2010)

\begin{abstract}
Topographically flat, single crystal silicon supersaturated with the chalcogens $\mathrm{S}$, Se, and Te was prepared by ion implantation followed by pulsed laser melting and rapid solidification. The influences of the number of laser shots on the atomic and carrier concentration-depth profiles were measured with secondary ion mass spectrometry and spreading resistance profiling, respectively. We found good agreement between the atomic concentration-depth profiles obtained from experiments and a one-dimensional model for plane-front melting, solidification, liquid-phase diffusion, with kinetic solute trapping, and surface evaporation. Broadband subband gap absorption is exhibited by all dopants over a wavelength range from 1 to 2.5 microns. The absorption did not change appreciably with increasing number of laser shots, despite a measurable loss of chalcogen and of electronic carriers after each shot. (C) 2010 American Institute of Physics. [doi:10.1063/1.3415544]
\end{abstract}

\section{INTRODUCTION}

There has been much interest recently in the subband gap optical ${ }^{1-7}$ and optoelectronic ${ }^{3}$ properties of chalcogensupersaturated silicon. These materials have been prepared by laser-structuring, ${ }^{1-4}$ laser incorporation, ${ }^{7}$ and ion implantation and pulsed laser melting., 5

During high-dose ion implantation, the implanted region becomes amorphous, and this material shows negligible subband gap optical absorption. ${ }^{6}$ Pulsed laser melting with a nanosecond laser is a well-established method of returning the material to a crystalline state. Ion implantation, pulsed laser melting, and rapid solidification have been found to result in high quality single crystalline material with sulfur concentrations four orders of magnitude in excess of the equilibrium solubility limit. ${ }^{5}$ Furthermore, the material exhibits strong subband gap optical absorptance in the wavelength range 1000-2500 nm, with absorption coefficients exceeding $10^{4} \mathrm{~cm}^{-1}$. Although the absorption coefficient is very sensitive to dose ${ }^{6}$ and anneal temperature, ${ }^{5}$ diode characteristics are relatively insensitive to dose but very sensitive to anneal temperature. ${ }^{6}$ Here we report a study of the influence of the laser treatment, including the number of laser shots received by the material, on the optical properties and concentration-depth profile of S, Se, and Te doped Si layers.

\section{EXPERIMENT}

$300 \mu \mathrm{m}$ thick, double-side polished $p$-type $\mathrm{Si}(001)$ wafers, resistivity $5-25 \Omega \mathrm{cm}$, were ion implanted at room

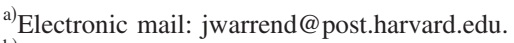

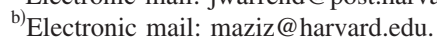

temperature with either $95 \mathrm{keV}{ }^{32} \mathrm{~S}^{+}, 176 \mathrm{keV}{ }^{80} \mathrm{Se}^{+}$, or 245 $\mathrm{keV}{ }^{130} \mathrm{Te}^{+}$, to doses of approximately $1 \times 10^{16} \mathrm{ions} / \mathrm{cm}^{2}$. All the samples were tilted by $\sim 7^{\circ}$ off the incident beam axis to minimize channeling. Pulsed laser melting was performed in ambient using a $\mathrm{XeCl}$ excimer laser beam (308 $\mathrm{nm}, 25 \mathrm{~ns}$ full width at half maximum, $50 \mathrm{~ns}$ total duration) at a fluence of $1.7 \mathrm{~J} / \mathrm{cm}^{2}$ (S implants) and $2.3 \mathrm{~J} / \mathrm{cm}^{2}$ (Se and Te implants). The spatially homogenized laser spot was approximately $3 \times 3 \mathrm{~mm}^{2}$. Time-resolved reflectivity using a low-power $\mathrm{Ar}^{+}$ion laser $(488 \mathrm{~nm})$ and a fast photodiode confirmed an optically flat surface was used to measure the melt duration. The laser fluence was always calibrated prior to melting the actual sample by measuring the melt duration of an untreated Si wafer under a given setting of the pulse energy and focusing optics, and comparing with numerical solutions of the one-dimensional (1D) heat equation that utilize the well-established optical and thermophysical properties of $\mathrm{Si}^{8,9}$

Secondary ion mass spectrometry (SIMS) was performed with a Physical Electronics 6650 quadrupole mass spectrometer using a $4 \mathrm{keV} \mathrm{Cs}^{+}$primary beam at an incident angle of $60^{\circ}$ from the surface normal and monitoring the ${ }^{28} \mathrm{Si},{ }^{32} \mathrm{~S},{ }^{80} \mathrm{Se}$, and ${ }^{130} \mathrm{Te}$ secondary ions. The depth scale was obtained by calibrating the sputter time with measured etch craters. The dopant concentrations were obtained by using measured doses from Rutherford backscattering spectrometry measurements (for the case of Se and Te) and by comparing the integrated yield under a SIMS profile at low sputtering rate with the expected dose from implantation (for the case of S).

Absorption data were obtained using a Hitachi U-4001 UV-VIS-NIR spectrophotometer equipped with an integrat- 


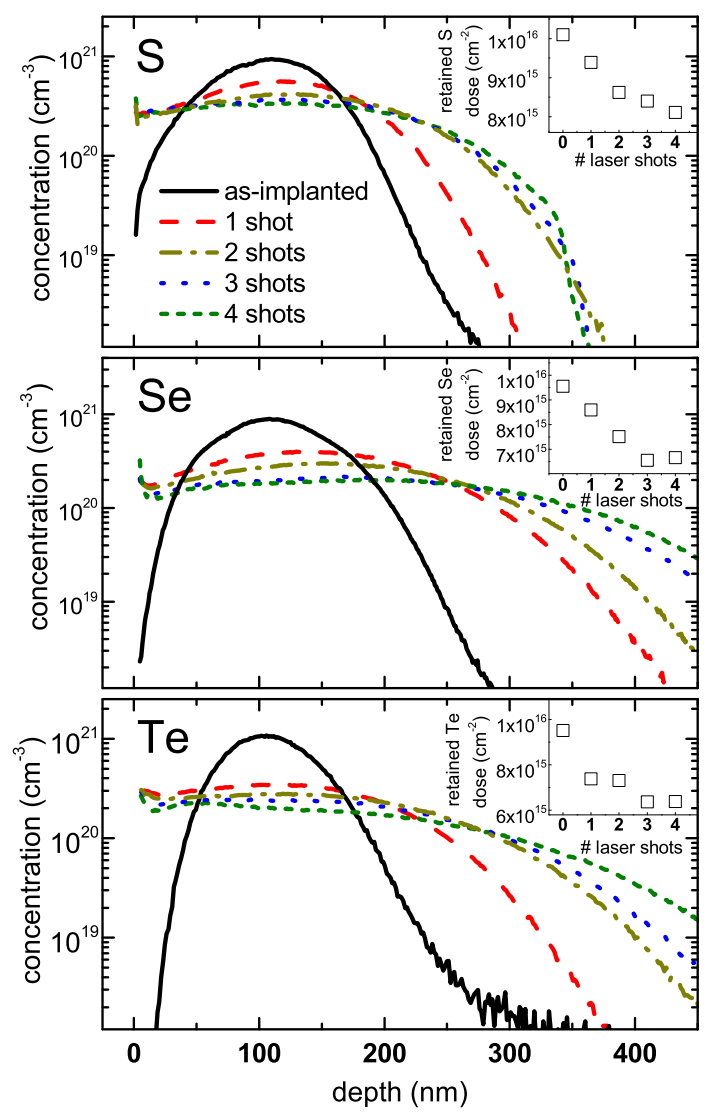

FIG. 1. (Color online) Experimental SIMS profiles of S, Se, and Te atoms (top, middle, bottom, respectively) in $\mathrm{p}-\mathrm{Si}(001)$, as a function of number laser shots. The insets show the total retained dose vs number of laser shots.

ing sphere detector. The spot was focused so that the entire beam was smaller than the laser melted area. The total absorptance $A$ was determined from the directly measured transmittance $T_{M}$ and reflectance $R_{M}$, according to $A=1$ $-T_{M}-R_{M}$. The reflectance spectra are calibrated using a gold mirror as a reference, and both the transmittance and absorptance spectra are smoothed by adjacent wavelength averaging. The measured reflectance, $R_{M}$, includes the effect of multiple reflections. When we use published reflectance data to estimate the reflectance at the front and back surface, the agreement between the estimated and measured values of $R_{M}$ is excellent. This indicates that reflectance of the front and back surface of our samples is close to that of an unimplanted silicon wafer. Therefore, we obtained the effective $\alpha d$ product, where $\alpha$ is the absorption coefficient and $d$ is the thickness of the absorbing layer, using $T_{M}$ and published reflectance data. ${ }^{10}$

Spreading resistance depth profiling (SRP) was performed on all samples (Solecon, Inc.). The measured resistivity $\rho$ was converted to carrier concentration $n$ using $n$ $=1 /[e \rho \mu]$ with $e$ the electron charge and $\mu$ the electron mobility. The model of Kaiblinger-Grujin et al. ${ }^{11}$ was used to calculate the chalcogen concentration-dependent mobility using the SIMS data to provide the atomic profiles.

\section{RESULTS AND DISCUSSION}

Figure 1 shows the calibrated SIMS concentration-depth
TABLE I. Parameters used in diffusion calculations and fitting of SIMS depth profiles.

\begin{tabular}{lcccc}
\hline \hline & & $v_{D}$ & $\begin{array}{c}D_{\text {liq }} \\
\left(\times 10^{-4} \mathrm{~cm}^{2} / \mathrm{s}\right)\end{array}$ & $\begin{array}{c}v_{\text {evap }} \\
(\mathrm{nm} / \mathrm{s})\end{array}$ \\
\hline $\mathrm{S}$ & No. of shots & $(\mathrm{m} / \mathrm{s})$ & 1.4 & 0.27 \\
$\mathrm{~S}$ & 1 & 1.0 & 1.4 & 0.054 \\
$\mathrm{~S}$ & 3 & 1.0 & 1.4 & 0.054 \\
$\mathrm{Se}$ & 4 & 1.0 & 1.4 & 0.21 \\
$\mathrm{Se}$ & 2 & 1.5 & 2.9 & 0.19 \\
$\mathrm{Te}$ & 3 & 1.5 & 2 & 0.27 \\
$\mathrm{Te}$ & 1 & 3.5 & 1 & 0.036 \\
$\mathrm{Te}$ & 3 & 3.5 & 3 & $\cdots$ \\
\hline
\end{tabular}

profiles for $\mathrm{S}, \mathrm{Se}$, and Te implants (top, middle, and bottom, respectively) for $0,1,2,3$, and 4 laser shots. The inset in each figure shows the total retained dose as a function of number of laser shots. The amount of retained chalcogen decreased by about $20 \%$ for the S sample and about $30 \%$ for the Se and Te samples after four laser shots.

To understand the evolution of the chalcogen concentration profile during laser melting, we used a numerical solution of the 1D diffusion equation with segregation at a moving boundary, which has been discussed previously. ${ }^{12}$ The validity of these heat flow calculations has been experimentally confirmed. ${ }^{8,9}$ Because the experimental profiles show significant chalcogen loss (Fig. 1), which we attribute to surface evaporation, we included this effect in the theoretical simulations in a simple way by assuming an evaporative flux given by $v_{\text {evap }} C_{0}(t)$, where $C_{0}(t)$ is the time-dependent chalcogen concentration in the liquid at the free surface and the surface evaporation velocity $v_{\text {evap }}$ is a unimolecular rate constant $^{13}$ analogous to the surface recombination velocity for carriers. Three input quantities are needed to fit the experimental SIMS profiles: $D_{\text {liq }}$, the liquid diffusivity of the dopant; $v_{D}$, the dopant diffusive velocity; and $v_{\text {evap. }}$ Experimental values for these parameters were not available, and therefore, they are treated as fitting parameters.

For $D_{\text {liq }}$ and $v_{D}$, we determined the value of these parameters that successfully reproduced for each laser pulse the deeper-than-peak region of each experimental SIMS profile (where evaporation effects should be negligible). These results are shown in Table I. For S, we found that for a single value of $D_{\text {liq }}$ and $v_{D}$, we could obtain good agreement for the evolution of all profiles. For Se and Te, a single $D_{\text {liq }}$ could not be used to fit the data for all profiles and so the values that give the best fit are shown in Table I. However, a single $v_{D}$ for Se and for Te did give a good fit to the deeper-thanpeak side of SIMS profiles. In cases where no values of $D_{\text {liq }}$ and $v_{D}$ could fit the data, no value is presented in Table I. We believe that the inability to obtain a good fit in such cases arises from problems with the calibration of the depth axis in the corresponding SIMS measurements.

For $v_{\text {evap, }}$, we determined the value that gave the best agreement with the shallower-than-peak region of each experimental SIMS profile, using the same best fit $D_{\text {liq }}$ and $v_{D}$ values shown in Table I. Figure 2 shows the agreement that was obtained for the evolution of the as-implanted $\mathrm{S}$ profile during the first laser shot. The agreement deeper than the 


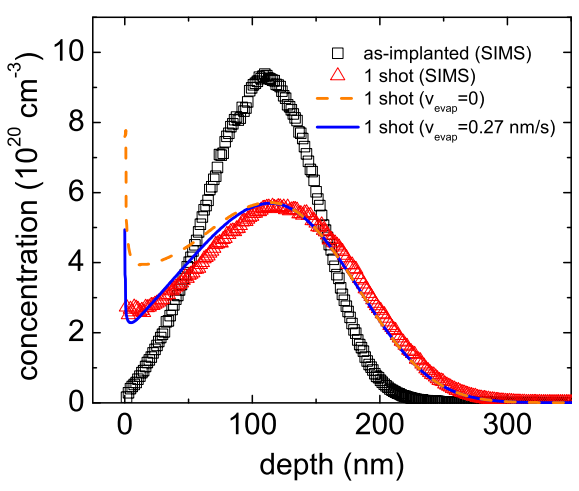

FIG. 2. (Color online) Calculations reproducing experimental SIMS profile evolved from as-implanted (squares) to 1-shot (triangles) using 1D diffusion calculation, with (dashed line) and without (solid line) surface evaporation included in the calculation.

impurity peak is good and is unaffected by the choice of $v_{\text {evap. }}$ Setting $v_{\text {evap }}=0.27 \mathrm{~nm} / \mathrm{s}$ gives reasonably good agreement with the entire SIMS profile, whereas $v_{\text {evap }}=0$, corresponding to no surface evaporation, gives poor agreement. Table I also shows the best fit value of $v_{\text {evap }}$ for each profile for S, Se, and Te samples; each value has an uncertainty of about $20 \%$ due to noise in the experimental data.

Figure 3 shows the measured transmittance $\left(T_{M}\right)$ and reflectance $\left(R_{M}\right)$. The panels correspond to S-, Se-, and Teimplanted samples, respectively, and each plot shows $T_{M}$ and

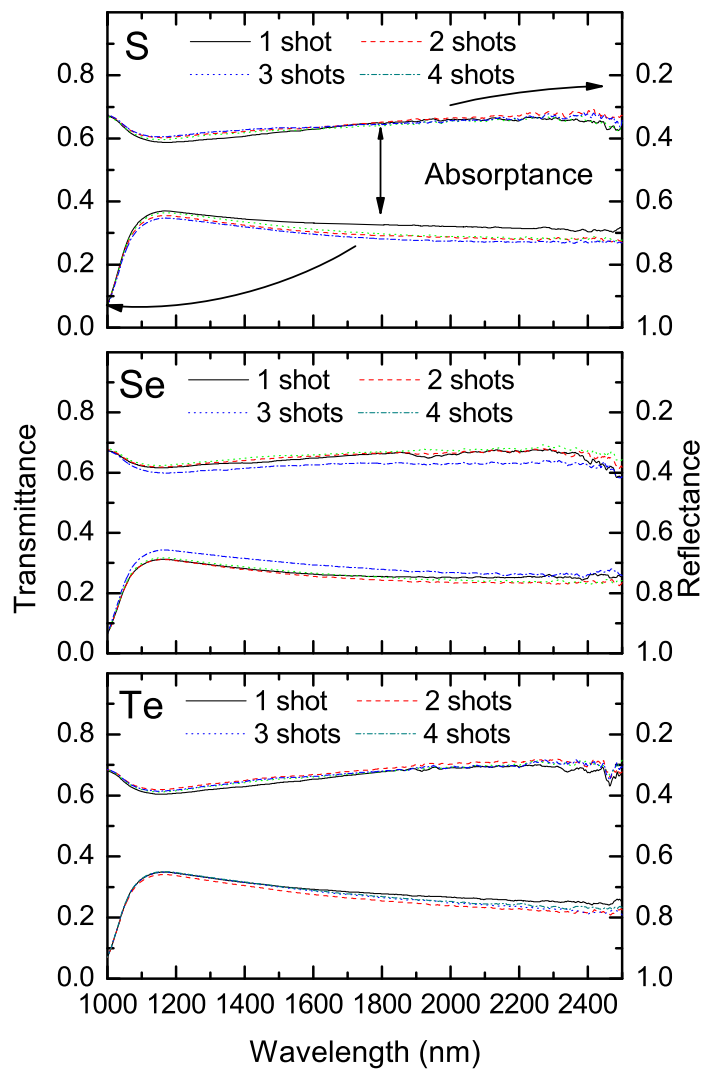

FIG. 3. (Color online) Reflectance (top curves-right axis, values plotted in reverse) and transmittance (bottom curves-left axis) for S, Se, and Te dopants (top, middle, and bottom panels) at varying number of laser shots. The vertical distance between a given reflectance and transmittance measurement is equal to the absorptance $A$.

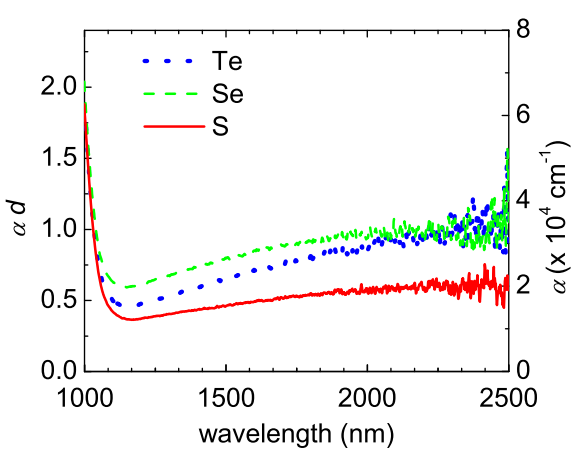

FIG. 4. (Color online) $\alpha d$ product (left axis), absorption coefficient (right axis) for $\mathrm{S}, \mathrm{Se}, \mathrm{Te}$ dopants, four laser shots. Absorbing layer thickness $d$ is approximated at $300 \mathrm{~nm}$ for all samples.

$1-R_{M}$ for samples that have received $1,2,3$, and 4 laser shots. The absorptance $A$ is therefore the vertical distance between the $R_{M}$ and $T_{M}$ curves. These are the same samples on which SIMS analysis described above was performed. In Fig. 4, we plot the $\alpha d$ product for S, Se, and Te implanted samples irradiated with four laser shots. The right axis shows the value of the absorption coefficient assuming a doped layer thickness $d=300 \mathrm{~nm}$.

Several features in these spectra are noteworthy. First, the absorption is reasonably uniform across the portion of the IR that is investigated here, but rises slightly with increasing wavelength. We have previously reported such behavior for S-doped samples. ${ }^{5}$ Second, the change in the reflectance, transmittance, and $\alpha d$ product with increasing number of shots is within the uncertainty of the measurement. We attribute this in part to differences in the samples themselves, arising from shot-to-shot variations in intensity delivered by each laser shot. To eliminate the effect of sample to sample variation, a single spot on a $\mathrm{Si}: \mathrm{S}$ sample was repeatedly irradiated and the $\alpha d$ product measured in the spectrophotometer. At $2000 \mathrm{~nm}$, the $\alpha d$ product increased by about $20 \%$ between the first and fourth shot. However, the increase appeared to saturate, as the difference between 3 and 4 shots is negligible.

Based on our previous observation that the absorptance depends on the chalcogen concentration, we might have expected to observe a more significant change in absorptance with increasing number of laser shots. ${ }^{5,14}$ It is conceivable that the laser heating may alter the doping concentrationdepth profile in a way that deviates from the (atomic) chalcogen concentration profile. To investigate this, we performed SRP measurements on all samples. The mobilities so obtained give excellent agreement with the values obtained from previous Hall measurements, as shown in Table II, for the $S$ dose $\left(1 \times 10^{16}\right.$ atoms $\left./ \mathrm{cm}^{2}\right)$ used in this current study, and reasonably good agreement at lower doses. ${ }^{6}$ Figure 5 shows the carrier concentration-depth profiles for 1-4 laser shots for each dopant. For all dopants, the carrier concentration shows a decrease with increasing number of shots. This decrease occurs over the full depth profile but is highest at the impurity peak. Comparing the carrier profiles in Fig. 5 with the SIMS atomic profiles in Fig. 1 reveals that (i) the carrier concentrations are considerably lower than the atomic concentrations (by a factor of at least 3 for $\mathrm{S}$ and $\mathrm{Te}$, and 
TABLE II. Comparison of calculated and measured mobilities for $\mathrm{S}$.

\begin{tabular}{cccc}
\hline \hline $\begin{array}{c}\text { Dose } \\
\left(\mathrm{cm}^{-2}\right)\end{array}$ & $\begin{array}{c}\mathrm{N}_{D}{ }^{\mathrm{a}} \\
\left(\mathrm{cm}^{-3}\right)\end{array}$ & $\begin{array}{c}\text { Hall mobility } \\
\left(\mathrm{cm}^{2} / \mathrm{V} \mathrm{s}\right)\end{array}$ & $\begin{array}{c}\text { Calculated mobility } \\
\text { (Kaiblinger-Grujin } \\
\text { formula) } \\
\left(\mathrm{cm}^{2} / \mathrm{V} \mathrm{s}\right)\end{array}$ \\
\hline $1 \times 10^{16}$ & $2.3 \times 10^{20}$ & 61 & 57 \\
$3 \times 10^{15}$ & $7.7 \times 10^{19}$ & 69 & 75 \\
$1 \times 10^{15}$ & $2.3 \times 10^{19}$ & 200 & 95 \\
\hline \hline
\end{tabular}

${ }^{\mathrm{a}}$ Reference 6.

more than an order of magnitude for Se) and (ii) after four laser shots the carrier concentration drops somewhat faster than the atomic concentration for all chalcogen dopants. This indicates that the fraction of electrically inactive to active chalcogen increases with increasing number of laser shots, but we cannot say conclusively whether the electrically active species are being lost to preferential ablation or because laser melting leads to the electrical deactivation of some of the chalcogen.

As we have previously reported, as-implanted samples show no subband gap absorption, ${ }^{5}$ and spreading resistance measurements of as-implanted samples are indistinguishable from those made on bulk wafers. This would suggest that the laser-induced crystallization must cause the chalcogen species to be optically active. Possible reasons include that the optically active species is either an isolated chalcogen atom on a particular site, a cluster containing a single chalcogen atom and one or more Si self-interstitials or impurities such as oxygen, ${ }^{15}$ or a cluster containing more than one chalcogen

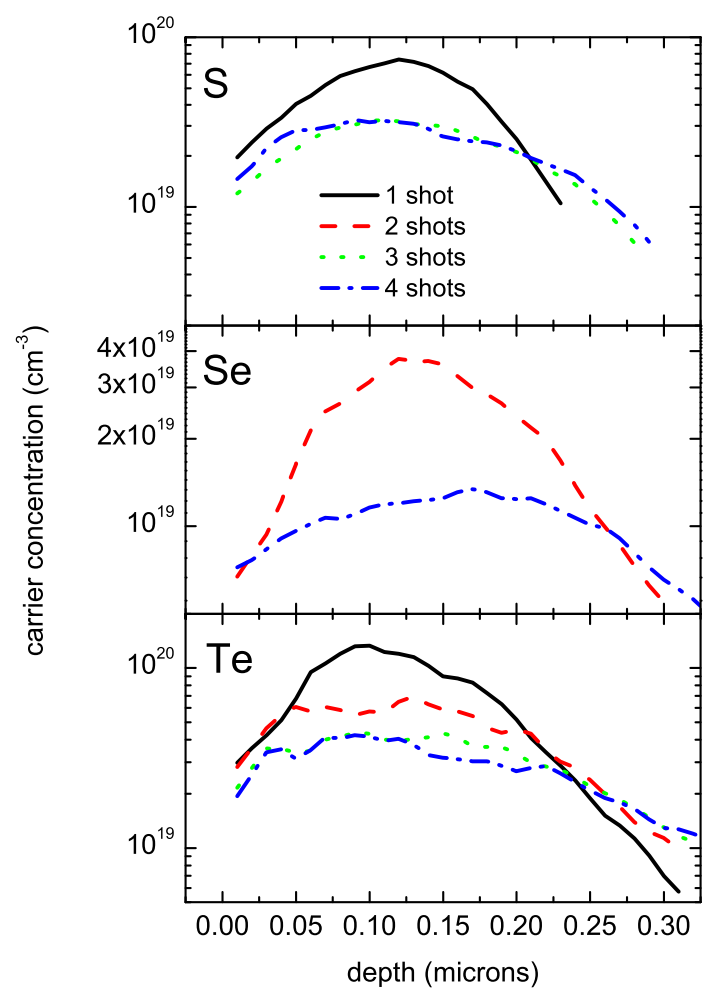

FIG. 5. (Color online) Carrier concentration calculated from spreading resistance profiles. Because SIMS data was used to calculate the mobility, profiles for which the SIMS data was deemed unreliable are omitted. atom. ${ }^{16}$ If the active species is an isolated chalcogen atom on a particular site, and clusters are not optically active, then we expect that optical absorption will increase in proportion to chalcogen concentration, ${ }^{15}$ unless the Fermi level moves significantly with chalcogen concentration. The behavior of an impurity-containing cluster containing a single chalcogen atom may be similar to that of an isolated chalcogen monomer, but the multishot behavior may be different depending on the nature of the cluster and the concentration of the impurity. SIMS of 4-shot Si:S samples showed an oxygen concentration of $2.1 \times 10^{19}$ atoms $/ \mathrm{cm}^{3}$ at the depth of the $\mathrm{S}$ peak, which is about $6 \%$ of the $\mathrm{S}$ concentration at that depth. The concentration of multichalcogen clusters should increase superlinearly with overall chalcogen concentration and, if their concentration is significant, this should be reflected in a sublinear dependence of the single-chalcogen-atom-bearing cluster concentration with overall chalcogen concentration. In this case, the absorption coefficient would display superlinear behavior if the optically active defect is a cluster containing more than one chalcogen atom and sublinear behavior if the optically active defect is a cluster containing only one chalcogen atom. It is also possible that clusters above a certain size are optically inactive; if this is the case, then over some range of concentration, the absorption should show sublinear behavior with increasing concentration.

To help further understand the relationship between atomic and electrical dopant concentrations and light absorption, we performed an experiment to measure the absorption coefficient versus depth for 1-shot and 4-shot Si:S samples. Reactive ion etching using an $\mathrm{Ar} / \mathrm{SF}_{6}$ plasma at a pressure of 10 mTorr was used to controllably remove a layer of material and profilometry was performed afterwards to identify the thickness of the layer that had been removed. Profilometry showed that the surface roughness was much smaller than the wavelengths being measured, and we, therefore, assume that the reflectance does not change as a result of the etching process. For each etch $n$, we obtained the absorption coefficient $\alpha$ for each etched layer for the $\mathrm{S}$ case for 1 and 4 laser shots. The average value of $\alpha$ for each layer was taken for photon energies between 0.6 and $1.1 \mathrm{eV}$, and is shown as a function of depth in Fig. 6. Data from two separate experiments are shown. The $\alpha$ for both the 1 shot and 4 shot samples are reasonably close at all depths, which is consistent with the earlier observation that the total absorptance does not change significantly with number of laser shots. In particular, even though there is $67 \%$ more $\mathrm{S}$ at the peak of the $S$ profile in the 1-shot sample compared with the 4-shot sample, the values of $\alpha$ for these samples differ by only a few percent. The inset shows $\alpha$ versus the average $\mathrm{S}$ concentration in each layer and indicates a general trend, whereby $\alpha$ increases with increasing $S$ concentration up to about 4 $\times 10^{20}$ atoms $/ \mathrm{cm}^{3}$, and does not increase further at higher $\mathrm{S}$ concentrations. The simplest explanation for this sublinear behavior is that the optically active species is either an isolated chalcogen atom on a particular site or a cluster containing a single chalcogen atom. However, we cannot rule out the possibility that optical absorption comes from a distribution of cluster sizes containing multiple chalcogen atoms, with a distribution of absorption cross sections. 


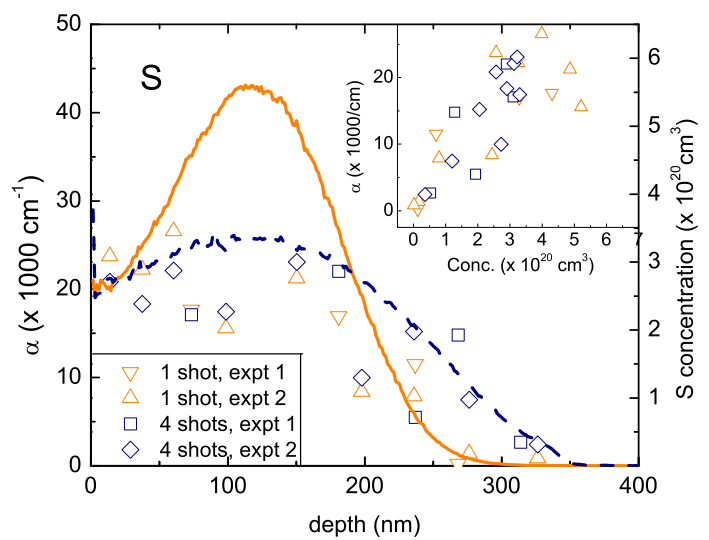

FIG. 6. (Color online) Average absorption coefficient for different layers obtained by sequential etching and spectrophotometry in 1-shot and 4-shot Si:S samples (left-hand axis). The atomic concentration-depth profiles measured by SIMS are shown (right-hand axis) for reference (1-shot, solid curve; 4-shot, dashed curve). The inset shows the average absorption coefficient as a function of the average $\mathrm{S}$ concentration in each layer.

\section{SUMMARY}

We have shown that doping with $\mathrm{S}$, Se, and Te chalcogen impurities in excess of the solubility limit can result in subband gap optical absorption in silicon over the wavelength range $1.0-2.5 \mu \mathrm{m}$. The chalcogens were introduced by ion implantation; pulsed laser melting was used to remove disorder and optically activate the chalcogen dopant in silicon. Numerical solutions to the 1D heat equation and the 1D diffusion equation were used to simulate the atomic chalcogen profile evolution during laser melting. Results indicated the following: (i) the atomic profiles following laser melting can be successfully modeled with simulations based on heat flow calculations with a constant liquid diffusivity and dopant diffusive velocity for each chalcogen, and a decreasing surface evaporation velocity with increasing number of laser shots for all chalcogens. (ii) Comparison of the SIMS atomic profiles with electrical profiles of the dopant indicated that only a small fraction of dopants was electrically active. (iii) The magnitude of absorption for all chalcogens did not change significantly with increasing laser shots over a large wavelength range despite the fact that the atomic $2 \mathrm{D}$ sheet concentrations dropped by about $15 \%$ from 1-shot to 4-shot laser processing and the electrical $2 \mathrm{D}$ sheet concentration dropped by between $45 \%$ and $75 \%$. (iv) There is not a strong correlation of depth profiles of the optical activity (absorption coefficient) with atomic and carrier concentration-depth profiles. This suggests that not all the chalcogen concentration is equally active optically and that some of the chalcogen concentration had clustered, with some of these clusters having different optical activity than nonclustered chalcogen.

\section{ACKNOWLEDGMENTS}

The authors gratefully acknowledge helpful conversations with D. Recht, M. Winkler, J. Carey, and E. Mazur. One of the authors (M.T.) acknowledges the financial support of the Fulbright Program. This research was supported in part by the U.S. Army ARDEC under Contract No. W15QKN-07P-0092.

${ }^{1}$ C. Wu, C. H. Crouch, L. Zhao, J. E. Carey, R. Younkin, J. A. Levinson, E. Mazur, R. M. Farrell, P. Gothoskar, and A. Karger, Appl. Phys. Lett. 78, 1850 (2001).

${ }^{2}$ C. H. Crouch, J. E. Carey, J. M. Warrender, E. Mazur, M. J. Aziz, and F. Genin, Appl. Phys. Lett. 84, 1850 (2004).

${ }^{3}$ J. E. Carey, C. H. Crouch, M. A. Sheehy, M. Shen, C. M. Friend, and E. Mazur, Opt. Lett. 30, 1773 (2005).

${ }^{4}$ M. A. Sheehy, L. Winston, J. E. Carey, C. M. Friend, and E. Mazur, Chem. Mater. 17, 3582 (2005).

${ }^{5}$ T. G. Kim, J. M. Warrender, and M. J. Aziz, Appl. Phys. Lett. 88, 241902 (2006).

${ }^{6}$ M. Tabbal, T. Kim, J. M. Warrender, M. J. Aziz, B. L. Cardozo, and R. S. Goldman, J. Vac. Sci. Technol. B 25, 1847 (2007).

${ }^{7}$ M. A. Sheehy, B. R. Tull, C. M. Friend, and E. Mazur, Mater. Sci. Eng., B 137, 289 (2007).

${ }^{8}$ M. J. Aziz, C. W. White, J. Narayan, and B. Stritzker, in Energy BeamSolid Interactions and Transient Thermal Processing, edited by V. T. Nguyen and A. G. Cullis (Editions de Physique, Paris, 1985), p. 231-236. ${ }^{9}$ R. Reitano, P. M. Smith, and M. J. Aziz, J. Appl. Phys. 76, 1518 (1994).

${ }^{10}$ Handbook of Optical Constants of Solids, edited by, by E. D. Palik, (Academic, New York, 1985).

${ }^{11}$ G. Kaiblinger-Grujin, T. Grasser, and S. Selberherr, in Simulation of Semiconductor Processes and Devices, edited by K. D. Meyer and S. Biesemans (Springer, Leuven, 1998), p. 312-215.

${ }^{12}$ D. E. Hoglund, M. O. Thompson, and M. J. Aziz, Phys. Rev. B 58, 189 (1998).

${ }^{13}$ T. Kim, K. Alberi, O. D. Dubon, M. J. Aziz, and V. Narayanamurti, J. Appl. Phys. 104, 113722 (2008).

${ }^{14}$ I. Umezu, S. Charnvanichborikarn, A. Kohno, J. M. Warrender, J. S. Williams, M. J. Aziz, M. Tabbal, D. Papazoglou, Y. Chen, and X.-C. Zhang (unpublished).

${ }^{15}$ T. G. Brown, P. L. Bradfield, and D. G. Hall, Appl. Phys. Lett. 51, 1585 (1987).

${ }^{16}$ T. G. Brown and D. G. Hall, Appl. Phys. Lett. 49, 245 (1986). 